\title{
The Structure of Deception: Validation of the Lying Profile Questionnaire
}

\author{
Dominique Makowski ${ }^{1, *}$, Tam Pham ${ }^{1}$, Zen J. Lau ${ }^{1}$, Adrian Raine ${ }^{2}$, \& S.H. Annabel Chen ${ }^{1,3,4, *}$ \\ ${ }^{1}$ School of Social Sciences, Nanyang Technological University, Singapore \\ ${ }^{2}$ Departments of Criminology, Psychiatry, and Psychology, University of Pennsylvania, Philadelphia, Pennsylvania, USA \\ ${ }^{3}$ Centre for Research and Development in Learning, Nanyang Technological University, Singapore \\ ${ }^{4}$ Lee Kong Chian School of Medicine, Nanyang Technological University, Singapore
}

\begin{abstract}
The conceptualization of deception as a dispositional trait is under-represented in the literature. Despite scientific evidence supporting the existence of individual differences in lying, a validated measure of dispositional deception is still lacking. This study aims to explore the structure of dispositional deception by validating a 16-item questionnaire to characterize individuals' lying patterns. The final sample included 716 participants (Mean age $=25.02$; $55.87 \%$ females) who were recruited via posters, flyers, and online social media platforms in Singapore. Our findings suggested four distinct latent dimensions: frequency, ability, negativity, and contextuality. We established the convergent validity of our measure by showing significant relationships with social desirability, malevolent traits, cognitive control deficits, normal and pathological personality traits, as well as demographic variables such as sex, age, and religiosity. Overall, the present study introduced a general framework to understanding deception as a dispositional trait.
\end{abstract}

Keywords: deception, lying, questionnaire, psychopathy, personality Word count: 7981

The scientific study of deception and lying has had a turbulent history, nourishing key developments in ethics and philosophy, and sometimes being (mis)used for forensic or political purposes. Although often put at the forefront of public attention through a criminological or psychopathological lens, the topic remains at the crossroads of different fields even within psychology. For example, although deception is a common phenomenon, practiced by virtually all (Kashy \& DePaulo, 1996), the emphasis has often been placed on lie detection or pathological cases. Thus, it seems that a general framework for understanding this phenomenon at the population level is still lacking.

Using an integrative framework, we propose to conceptualize deception as the process of achieving an inaccurate experience of a piece of information in relation to its objective qualities. This definition is neutral to the nature of the object and its cause, i.e., not limited for instance to verbal information (visual illusions could be interpreted as a form

Correspondence concerning this article should be addressed to Dominique Makowski, HSS 04-18, 48 Nanyang Avenue, Singapore. E-mail: dmakowski@ntu.edu.sg of sensory deception), humans (deceptive behaviours being documented in other species; e.g., Hirata, 1986; Waal, 2005) nor specific purposes (e.g., being necessarily beneficial for the deceiver, as suggested by Bond \& Robinson, 1988). As such, the study of this form of reality-bending could benefit from being placed within a larger framework of the sense of reality and its naturally connected neurocognitive functions such as perception, emotions or consciousness (Makowski et al., 2017; Makowski, 2018; Riva et al., 2007; Seth et al., 2012).

Lying is a form of intentional deception. It includes the creation and delivery of information that is believed to be inaccurate, with the aim of making it believed to be accurate (note that both deception and lying are used interchangeably in the context of this study). While lying is often studied as an act, involving but not limited to its production or reception, lying could also be understood and investigated as a dispositional trait, i.e., as a metastable characteristic of personality. In fact, one of the motivations supporting this perspective is the emergence of evidence supporting the existence of interindividual variability in lying.

Since lying can be described as a common and potentially universal phenomenon (Kashy \& DePaulo, 1996), one naive hypothesis could be that individual differences are less rele- 
vant than situational factors (Aquino \& Becker, 2005). However, empirical evidence has recently rebutted the assumption of ubiquitous lying behaviors, suggesting that the extent to which people engage in lying varies considerably from one individual to the next (Gozna et al., 2001; Kashy \& DePaulo, 1996). For instance, differences have been observed between participants in the frequency of lying, their (perceived) ability to tell and detect lies, the emotions associated with lying (e.g., guilt) or the moral attitude toward it (Serota et al., 2010; Serota \& Levine, 2015). Importantly, these patterns have been shown to be related to other interindividual variables Jensen et al. (2004). In spite of this evidence, most experimental research on deception has overlooked trait-like interindividual variability, and has instead focused on the behavioral and neural correlates of lying and lie detection. Critically, it is possible that the absence of such control of traitdeception in experimental studies is related to a lack of validated questionnaires made to assess individual variations in lying.

However, using self-report questionnaires as a measure of deception inherently begs the question of whether results may be contaminated by response bias and distorted perceptions in self assessment. Although the issue of deception assessment using self-report tools being beyond the scope of this study, past studies have demonstrated some level of support for its suitability. Self-reported lying frequencies have been shown to converge with respondents' estimations of others' lying tendencies (Serota et al., 2010), as well as real life dishonesty in the lab (i.e., cheating on a task, Halevy et al., 2014a). A methodologically rigorous approach is also crucial in eliminating any potential caveats. This includes accounting for social desirability to adjust for each subject's bias (Fisher \& Katz, 2000; Serota et al., 2010) and encouraging accurate reporting through guaranteeing data anonymity (Serota \& Levine, 2015) and providing a clear definition of lying in the questionnaire (Serota et al., 2010). These additional measures are closely followed in the present study and will be elaborated on in later sections.

Indeed, while "lie scales" are common in psychometric research, they often refer to social desirability measures (e.g., the LIE subscale in the Eysenck Personality Questionnaire, Eysenck \& Eysenck, 1975) rather than to validated questionnaires reliably measuring different aspects of deception. Existing developments in lie scales are also often limited to measures that are created and used in studies targeting deception as a dependent or independent variable (as opposed to an exploration of the dimensionality to trait-deception). For instance, Azizli et al. (2016) assessed the tendency to lie in a high-stake deception using the in-house created Propensity To Lie Questionnaire. The first part of this questionnaire included questions about the general subjective tendency to lie, while the second part includes items pertaining to two short scenarios describing hypothetical lying situations. The findings reported a relationship between the tendency to lie and antisocial tendencies (Machiavellianism, narcissism, and psychopathy). Unfortunately, this study did not report normative data nor a factor structure analysis of the questionnaire. Similarly, El Haj et al. (2018) created a five-item scale, adapted from the Impression Management subscale of the Balanced Inventory of Desirable Responding (BIDR, Delroy L. Paulhus, 1991), assessing the tendency to lie (or rather, to impress others), and showed that people with a higher trait tendency to lie have a higher ability to remember to whom they have told a piece of information. More in line with our dimensional approach to trait-deception, Zvi and Elaad (2018) developed the Lie-Truth Ability Assessment Scale to measure different facets related to lying, such as the ability to tell lies, to perceive lies, to tell the truth, and also to believe others. Although the factor structure and internal reliability of the scale were not thoroughly tested, the study reported a positive relationship between lying ability and narcissism. Nonetheless, the authors emphasize the need to develop a reliable and valid lie scale.

Using a different approach, Serota and Levine (2015) investigated how participants can be grouped into different "profiles" based on the number of lies they tell per day. They suggested the existence of two distinct groups, everyday liars, the majority of the general population, and prolific liars, who possess a significantly higher tendency to lie and whose dishonest behaviors are often associated with serious matters. This is in line with the studies suggesting that manipulativeness, sociability, anxiousness, and impression management are features related to a stronger self-reported lying ability (Elaad \& Reizer, 2015; Gozna et al., 2001; Kashy \& DePaulo, 1996; Panasiti et al., 2011). Unfortunately, methodological limitations of the study (the absence of statisticallydriven cluster analysis) underline the necessity to further investigate this interesting profile perspective.

One of the challenges for validating a lying questionnaire is the identification of relevant related constructs to assess convergent validity. Although the literature on trait-deception described above is rather sparse, experimental and observational research investigating how deceptive behaviors relate to other inter-individual characteristics can be used as a starting point to establish specific and testable hypotheses. For instance, a large body of research has investigated how lying relates to normal personality traits (Gozna et al., 2001), with evidence suggesting that extraversion is related to a higher lying frequency (Weiss \& Feldman, 2006), contrary to conscientiousness which tends to be related to honesty (Gillath et al., 2010). Perceived ability to tell lies shares a positive relationship with extraversion and openness and a negative relationship with agreeableness (Elaad, 2018; Elaad \& Reizer, 2015; Kashy \& DePaulo, 1996). The hypothesized underly- 
ing link is that people with higher extraversion and openness are more likely to engage in social events, offering more opportunities to lie (Kashy \& DePaulo, 1996), which in turn boosts the frequency of - as well as their confidence in - lying. On the other hand, agreeable and conscientious individuals are less likely to lie (and arguably more honest about themselves), consequently describing themselves as being less skilled at it (Gillath et al., 2010).

Another personality dimension related to deception is narcissism (Zvi \& Elaad, 2018), a trait commonly found in several personality disorders and one of the facets of the socially malevolent personality profile coined as the "dark triad" (Delroy L. Paulhus \& Williams, 2002). Given that narcissists value power and are endowed with a grandiose sense of self, their primary motive to lie is for self-gain and selfenhancement Dike et al. (2005). Another possible mechanism at play could be the role of grandiosity in supporting self-deception in the context of negative feedback, which would subsequently facilitate lying to others (Uziel, 2014; Wright et al., 2015).

Naturally, deception is most commonly studied in relationship with antisocial and antagonistic traits, such as psychopathy (Hare \& Forth, 1985), which is positively correlated with lying frequency (Halevy et al., 2014b), with self-reported lying ability and with the tendency to lie without reason (Jonason et al., 2014). Neuroimaging evidence during a deception task has suggested that specific aspects of psychopathy, namely fearlessness and coldheartedness, were associated with lower activity in the orbitofrontal and temporal cortex, respectively (Fullam et al., 2009). These findings emphasize the potential role of social and emotional sensitivity and control in modulating deceptive behaviors.

More specifically, it is plausible that the relationship between higher-order antisocial traits and deception could be supported by lower-order processes, such as self-control which has been shown to be correlated with the "dark triad" (Jonason \& Tost, 2010). This is in line with theoretical and empirical evidence suggesting the implication of cognitive control in lying (Abe, 2009; Debey et al., 2012; Lee et al., 2009; Poletti et al., 2011), supporting processes such as decisionmaking, taking others' perspectives, maintaining consistency of the fabricated story or inhibiting previously learned content or true responses. This is consistent with neuroscientific findings underlining the role of prefrontal regions in deception (e.g., Christ et al., Oxford University Press, UK; Karim et al., 2010) as well as with individuals reporting greater cognitive effort when having to lie as compared to telling the truth (Vrij \& Semin, 1996). Nonetheless, as most of the evidence presented above was gathered in experimental settings, it remains unclear how these variables are related to dispositional deception.

Thus, this study aims primarily at investigating the factor and cluster structure of lying as a trait. To achieve these two respective aims, this study explores how questions about lying map onto latent factors related to deception, and whether groups of individuals emerge in the uncovered multidimensional space of deception facets. This will be accomplished through the validation of a short yet reliable questionnaire on the general population, which will allow future deception studies to account for the inter-individual variability in the natural disposition to lie.

In line with the evidence presenting deception as a phenomenon supported by a neurocognitively distributed network of processes, we hypothesized the questions related to deception to preferentially fit a multidimensional structure, composed of different distinct latent factors. Regarding convergent validity, we expect deception to be positively related to malevolent and antisocial traits, such as psychopathy, antagonism, and narcissism. Correspondingly, we also predict a negative link with benevolent traits, such as agreeableness or the recently defined "light triad" traits (Kaufman et al., 2019). A relationship is also expected with normal personality dimensions, such as extraversion, openness and honestyhumility, as well as to traits related to potential deficits of cognitive control, such as impulsivity (Enticott et al., 2006; Fino et al., 2014) and emotion regulation (Kohn et al., 2014; Makowski, Sperduti, et al., 2019; Ochsner \& Gross, 2005; Sperduti et al., 2017). Notably, beyond its use as a mere proxy of cognitive control, difficulties in emotion regulation might impact lying in another way, as the engagement in deception might be dependent on one's ability to cope with the emotional states related to lying - e.g., stress - and its consequences - e.g., guilt or shame (Arndt et al., 2013; Carlson \& Wang, 2007).

Another aspect of the relationship between emotions and cognitive processes (such as decision making), understudied in the context of deception, is interoception, which refers to one's sensitivity to internal signals and bodily states (Füstös et al., 2012; Garfinkel et al., 2015; Kever et al., 2015). Indeed, decision-making research has shown that individuals with higher interoceptive awareness are less likely to make risky decisions (Dunn et al., 2010; Furman et al., 2013). This is in accordance with the somatic marker hypothesis, in that the accurate detection of physiological arousal guide the use of such interoceptive feedback to make safe, reasoned decisions (Bechara \& Damasio, 2005). By extension, since lying could be perceived as a risky behaviour that increases bodily arousal (e.g., Turck \& Miller, 1985), we expect interoception to be related to lying behaviour (for instance, lying frequency or ability). 


\section{Methods}

The study plan was preregistered (https://osf.io/3kv7f). In the spirit of open and honest science, the raw data, as well as the entire analysis script as Supplementary Materials 1 (including details and additional analyses) can be found at https://github.com/DominiqueMakowski/2020structure. An interactive web application to compute the scores of the questionnaire is available at https://neuropsychology.shinyapps. io/proflier.

\section{Participants}

One thousand and eleven participants from the general population were initially recruited via posters and flyers, as well as online social media platforms (e.g., Facebook). Inclusion criteria included residing in Singapore, as well as an absence of neurological and psychiatric history. Participants were reimbursed 5 Singapore Dollars in cash or vouchers upon survey completion. The study was approved by the Institutional Review Board (Reference Number: IRB-2019-02026) of Nanyang Technological University (NTU).

Due to the presence of a monetary incentive, as much as to the nature of the investigated construct, a strict procedure was used in order to ensure and maximize data quality. 5 participants were excluded due to missing data, followed by 141 participants with a completion time outside the $90 \%$ percentile $[<10.95 \mathrm{~min}$ and $>61.94 \mathrm{~min}$ ]. In addition to completion time (which is considered as the best indicator of data quality, Leiner, 2013), we identified multivariate outliers based on a composite outlier score (see the check_outliers function in the performance $\mathrm{R}$ package, D. Lüdecke et al., 2019) obtained via the joint application of multiple outliers detection algorithms (e.g., Mahalanobis distance, Invariant Coordinate Selection, or Local Outlier Factor; see D. Lüdecke et al., 2019 for the full description). This led to the exclusion of 149 participants that were classified as outliers by more than half of the methods used.

The final sample included 716 participants (Mean age = 25.02, $\mathrm{SD}=7.07$, range $=[16.55,73.51] ; 55.87 \%$ females; Mean education in years relative to high school completion $=3.54, \mathrm{SD}=1.95$, range $=[-7,10]$; Mean Religious Engagement $=3.82, \mathrm{SD}=3.09$, range $=[0,10] ;$ Mean Religiosity $=$ $4.22, \mathrm{SD}=2.97$, range $=[0,10]$; Median monthly income per household per capita $=$ SGD2,000, MAD $=$ SGD1482.60).

\section{Procedure}

Participants completed an online questionnaire created via the Qualtrics@platform. After informed consent was obtained, participants responded to items on visual analog scales or Likert scales (adapted to screen size). Items from 9 existing questionnaires, as well as our Lying Profile Questionnaire (LIE), were included (see below). The presentation order of these 10 inventories was randomized. Note that while items within the LIE inventory were presented in a randomized order, all existing questionnaires were presented following their original validation, and scores for their dimensions were calculated accordingly (by averaging or summing, see Supplementary Materials 1). Participants then responded to questions about demographics at the end of the questionnaire. A transcript of the complete survey presentation is available in Supplementary Materials 2.

\section{Measures}

Lying. Based on the theoretical literature and the existing scales on lying, we outlined 3 general domains related to lying relevant for a questionnaire; lying frequency, ability, and accompanying features, such as motives and reactions. From there, we developed an initial pool of items later refined for face validity, ambiguous wording, jargon, or poor phrasing in focus group discussions. The final Lying Profile Questionnaire (LIE) included 44 items phrased in statements such as "I lie more often than most people do" or "I find lying difficult," presented on visual analog scales with "Disagree" and "Agree" as the two extremities. While the LIE items required relative subjective judgments, we also included two questions pertaining to the Absolute Frequency of lying (asking how many lies one tells per day and per week). We averaged these two items into one score expressed in lies/day.

We adapted our self-report lie measures in accordance with the methodology used by Serota et al. (2010) to encourage accurate reporting. Prior to recording participants' responses, we provided a definition of lying as encompassing the intent to deceive. This is accompanied with descriptions of different types of lies, making sure to eliminate any instances of disparagement in these presentations. Participants are thus encouraged to consider lies of different magnitude, content (to emphasize that half-truths are also lies), effectiveness, intent (i.e., told for prosocial or antisocial purposes), and consequences. The description is available in Supplementary Materials 2 (p. 21).

Social Desirability. The 16-item Balanced Inventory of Desirable Responding (BIDR-16) self-report questionnaire (Hart et al., 2015) was included to control for related biases in responding such as over-reporting positive traits and under-reporting lying tendencies. This scale includes 2 distinct dimensions, Self-Deceptive Enhancement (participants' deception of themselves with a tendency towards positive traits), and Impression Management (participants' intention to deceive for the sake of pleasing others). 
Psychopathy. The 58-item Triarchic Psychopathy Measure self-report questionnaire (TriPM, Christopher J. Patrick, 2010) was used to assess the 3 dimensions suggested by the triarchic model of psychopathy (Christopher J. Patrick et al., 2009), namely Boldness (fearlessness, social dominance, and the tendency to engage in adventure-seeking behaviors), Disinhibition (the lack of behavioral restrain, manifesting as impulsivity, disregard for social conventions, and aggression) and Meanness (the unempathetic and instrumental treatment of others).

Narcissism. The short version of the Five-Factor Narcissism Inventory Sherman et al. (2015) was used to measure 9 specific traits, namely Acclaim Seeking (preoccupation with achieving acclaim, status, and/or fame), Entitlement (expectations of special and self-serving treatment), Need for Admiration (excessive need for the admiration and approbation of others), Manipulativeness (a disposition to deceptively manipulate the feelings and/or opinions of others), Lack of Empathy (failure to be aware of, appreciate, or acknowledge the feelings of others), Indifference (lack of self-consciousness or self-doubt in response to criticism or rebuke), Thrill Seeking (excessive excitement-seeking that leads to high-risk behavior for the sake of thrills and excitement), Distrust (maladaptive low level of trust concerning the intentions and motivations of others), and Exploitativeness (a disposition for instrumental treatment of others, i.e., to exploit or take advantages of others).

Normal Personality. The 24-item measure of the Big-Six personality dimensions (Mini-IPIP6, Sibley et al., 2011) was used to measure the Big Six "normal" (as opposed to pathological) personality traits based on the HEXACO Personality Model (Ashton \& Lee, 2009), namely Extraversion (the tendency to engage in social behaviors such as exhibiting leadership and sociability), Openness (the extent to which one is open-minded in terms of imagination and curiosity), Agreeableness (how cooperative and tolerant one is of others, with individuals high on this trait often being perceived as warm, forgiving, and kind), Conscientiousness (being diligent, meticulous and organized during task execution), $\mathrm{Neu}$ roticism (the tendency to experience a persisting negative emotional state), and Honesty-Humility (being honest, sincere, and fair during social exchanges).

Pathological Personality. The 25 -item Personality Inventory for DSM-5 Brief Form (PID-5-BF) was used to assess 5 pathological personality traits (Al-Dajani et al., 2016; Hopwood et al., 2012), namely Negative Affect (the frequency and intensity of negative emotional experiences such as anxiety, anger, and depression), Detachment (social withdrawal and diminished affective experiences), Antagonism (manipulativeness, deceitfulness, callousness and hostility) Disinhibition (engagement in impulsive behaviors for immediate gratification), and Psychoticism (eccentric or incongruent behaviors and cognitions, such as hallucinations and delusions).

Light Triad. The 12-item Light Triad Scale (LTS, Kaufman et al., 2019) was used to measure prosocial and morally positive traits (as opposed to antisocial or antagonistic ones), including Faith in Humanity (the belief and trust that individuals are fundamentally good in nature), Humanism (the extent to which one places value on the dignity and worth of others), and Kantianism (the treatment of individuals as means to themselves rather than using them instrumentally).

Impulsivity. The 20-item Short UPPS-P Impulsive Behaviour Scale (Cyders et al., 2014; Whiteside \& Lynam, 2001) was used to measure 5 facets of impulsivity, namely Negative Urgency and Positive Urgency (one's propensity to act impulsively under negative and positive emotional states, respectively), Lack of Perseverance (the inability to focus on tasks of a boring or difficult nature), Lack of Premeditation (the propensity to act without thinking), and Sensation Seeking (the inclination towards partaking in novel and thrilling experiences).

Emotion Regulation. The 18-item Difficulty in Emotions Regulation Scale (DERS, Victor \& Klonsky, 2016) was used to measure 6 facets of emotion regulation deficits, namely Awareness (lack of recognition and appreciation of one's emotions), Clarity (difficulties in giving meaning to emotions), Goals (difficulties in engaging in goal-directed cognition and behavior when distressed), Impulse (lack of control when distressed), Non-Acceptance (unwillingness to accept certain emotional responses), Strategies (lack of access to strategies for feeling better when distressed).

Interoception. We used 11 items from the Multidimensional Assessment of Interoceptive Awareness, Version 2 (MAIA-2, Mehling et al., 2018) to specifically measure 2 facets of interoception, namely Noticing (the conscious awareness of bodily sensations), and Body Listening (he ability and tendency for active listening to the body for insight).

Demographic. Participants provided demographic information related to their Education (highest academic qualification achieved or the qualification they are currently pursuing), Sex, Age, and their socio-economic status (SES) which was operationalized as the average monthly household Income per capita. Additionally, two items related to religious Faith were presented on Likert scales, pertaining to how religious the participants perceive themselves to be and how actively engaged in religious activities they are.

\section{Data Analysis}

We started by investigating the factor structure of our initial set of items. We randomly split the study sample into 
a training set (60\%) and a test set (40\%). Exploratory Factor Analysis (EFA) was carried out with the training set to explore the scale's underlying factor structure and Confirmatory Factor Analysis (CFA) was performed with the test set to test the goodness-of-fit of the suggested factor structures. We then examined the cluster structure using k-means clustering and assessed the convergent validity with partial correlation analysis.

Data processing was carried out with $\mathrm{R}$ (R Core Team, 2019) and in particular the psych (Revelle, 2018) and the lavaan (Rosseel, 2012) packages, as well as the easystats ecosystem (Daniel Lüdecke et al., 2019; Makowski, Ben-Shachar, \& Lüdecke, 2019a). The raw data, as well as the full reproducible analysis script (along with complementary results and figures), are available in Supplementary Materials 1.

\section{Results}

\section{Factor Structure}

The 44 initial items were deemed suitable for factor analysis $\left(\mathrm{KMO}=0.93\right.$; Bartlett's test of sphericity $\chi^{2}(946)=9653.38$, $p<.001)$. The factor number exploration using the method agreement procedure (see the n_factors function in the parameters package, Makowski, Ben-Shachar, \& Lüdecke, 2019b) suggested 2 optimal factor solutions: four factors, and one latent factor, respectively accounting for $43.00 \%$ and $24.63 \%$ of variance of the dataset. Hence, we submitted the unique one-factor and four-factor models to Confirmatory Factor Analysis (CFA).

Table 1

CFA fit indices of the full four-factor model, one-factor model, hypothesized model, and the short-form four-factor models (containing 3, 4, and 5 loading items per dimension respectively).

\begin{tabular}{lrrrrrr}
\hline Model & AIC & BIC (adj.) & Chi2 & RMSEA & CFI & SRMR \\
\hline Four Factors: all items & 57650.70 & 57702.29 & 2142.547 & 0.068 & 0.821 & 0.089 \\
One Factor: all items & 59691.09 & 59739.39 & 4194.940 & 0.109 & 0.527 & 0.122 \\
Hypothesized: all items & 58197.46 & 58247.40 & 2695.311 & 0.081 & 0.742 & 0.097 \\
Four Factors: 3 items & 15296.48 & 15312.94 & 117.046 & 0.069 & 0.960 & 0.061 \\
Four Factors: 4 items & 20309.91 & 20330.76 & 223.804 & 0.065 & 0.949 & 0.061 \\
Four Factors: 5 items & 25408.64 & 25433.89 & 322.178 & 0.056 & 0.949 & 0.061 \\
\hline
\end{tabular}

The confirmatory factor analysis favoured the four-factor solution over the one-factor. We then compared the four-factor solution with the initial hypothetic model with which we built the scale, which favoured the four-factor model. Finally, we compared the full four-factor model (including all items) with short forms retaining only the 3,4 or 5 most loading items for each of the 4 dimensions. The 3 -items version outperformed all versions, including 5 -items and 4items. Nonetheless, as 3-items per construct is the bare minimum for adequate reliability, we decided to keep the second best performing version with 4 -items per factor, which also displayed excellent indices of fit (see Table 1).
Table 2

All initial item loadings from the Exploratory Factor Analysis (EFA). The final item selection (the 4 most loading items of each dimension) also has the corresponding regression coefficients from the Confirmatory Factor Analysis (CFA) model that were used to calculate individual scores (between brackets).

\begin{tabular}{|c|c|c|c|c|c|}
\hline Item & Label & Frequency & Ability & Negativity & Contextuality \\
\hline Q4 & I have a tendency to lie & $0.75[1.01]$ & 0.17 & -0.17 & 0.05 \\
\hline Q23 & I find it difficult to refrain myself from lying & $0.73[0.82]$ & 0.11 & -0.15 & $5.67 \mathrm{e}-03$ \\
\hline Q5 & I lie more often than most people do & $0.73[0.92]$ & 0.18 & -0.24 & $-8.04 \mathrm{e}-03$ \\
\hline Q1 & I lie frequently & $0.70[1.00]$ & 0.18 & -0.30 & 0.15 \\
\hline Q22 & I find myself lying without any reason & 0.68 & 0.06 & -0.14 & $-4.26 \mathrm{e}-04$ \\
\hline Q7 & I lie more than I think I should & 0.67 & 0.05 & $-8.44 \mathrm{e}-03$ & 0.11 \\
\hline Q6 & I lie more frequently than what I expect myself to & 0.65 & 0.10 & -0.08 & 0.11 \\
\hline $\mathrm{Q} 2$ & I lie in many situations & 0.61 & 0.18 & -0.34 & 0.09 \\
\hline Q26 & I enjoy lying & 0.59 & 0.23 & -0.24 & -0.03 \\
\hline Q8 & Others lie less often than I do & 0.53 & 0.14 & -0.11 & 0.02 \\
\hline Q29 & I lie whenever it's convenient & 0.49 & 0.18 & -0.18 & 0.25 \\
\hline Q21 & I have to try hard to avoid lying & 0.45 & -0.04 & 0.09 & -0.08 \\
\hline Q31 & I lie if it's the most direct way to get what I want & 0.44 & 0.12 & -0.12 & 0.31 \\
\hline Q28 & I feel satisfied when others believe my lie & 0.36 & 0.24 & -0.12 & 0.30 \\
\hline Q24 & It is easy to hold back from telling lies & -0.29 & 0.09 & 0.15 & 0.06 \\
\hline $\mathrm{Q} 10$ & I can lie well & 0.25 & $0.82[1.05]$ & -0.14 & 0.21 \\
\hline $\mathrm{Q} 9$ & I am a good liar & 0.30 & $0.75[1.00]$ & -0.17 & 0.18 \\
\hline Q18 & It is easy for me to make up clever lies & 0.25 & $0.73[0.87]$ & -0.11 & 0.16 \\
\hline Q14 & It is hard for others to detect my lies & 0.15 & $0.73[0.77]$ & -0.03 & 0.18 \\
\hline Q11 & I am good at deceiving others & 0.33 & 0.71 & -0.11 & 0.14 \\
\hline Q13 & Others can easily tell when I'm lying & 0.11 & -0.69 & 0.19 & -0.06 \\
\hline Q12 & I can lie effectively if I want to & 0.13 & 0.67 & -0.04 & 0.27 \\
\hline Q17 & I find lying difficult & -0.08 & -0.67 & 0.42 & -0.14 \\
\hline Q15 & I almost never get caught lying & 0.08 & 0.65 & -0.07 & 0.25 \\
\hline Q20 & I do not have to prepare much for a lie & 0.22 & 0.58 & -0.13 & 0.16 \\
\hline Q19 & I find it taxing to come up with a good lie & -0.06 & -0.49 & 0.36 & 0.07 \\
\hline Q27 & I feel tense whenever I have to lie & -0.03 & -0.49 & 0.44 & 0.04 \\
\hline Q16 & My lies often arouse suspicion from others & 0.28 & -0.46 & 0.11 & -0.13 \\
\hline Q41 & Lying is against my principles & -0.19 & -0.23 & $0.62[1.30]$ & -0.19 \\
\hline Q34 & I always avoid lying if I can & -0.41 & -0.05 & $0.57[0.86]$ & 0.06 \\
\hline Q44 & It is bad to lie & -0.13 & -0.21 & $0.55[1.07]$ & -0.17 \\
\hline Q25 & I feel guilty after lying & -0.08 & -0.30 & 0.54 [1.00] & -0.09 \\
\hline Q36 & I prefer to tell the truth even if it gets me into trouble & -0.20 & -0.12 & 0.46 & -0.13 \\
\hline Q35 & I would only lie if I have no other choice & -0.12 & 0.01 & 0.36 & 0.32 \\
\hline Q37 & I would never lie for trivial matters & -0.15 & -0.03 & 0.34 & 0.02 \\
\hline Q38 & I would never lie in serious contexts & -0.11 & -0.10 & 0.31 & -0.01 \\
\hline Q43 & It is okay to lie sometimes & 0.07 & 0.16 & -0.21 & $0.71[1.21]$ \\
\hline Q33 & I lie when necessary & 0.08 & 0.18 & -0.02 & $0.69[1.00]$ \\
\hline $\mathrm{Q} 42$ & It is acceptable to lie depending on the context & 0.02 & 0.25 & -0.10 & $0.62[1.03]$ \\
\hline Q39 & I would lie if something important was at stake & 0.02 & 0.18 & 0.05 & $0.47[0.78]$ \\
\hline $\mathrm{Q} 40$ & I would only lie if it is harmless & -0.06 & 0.10 & 0.09 & 0.46 \\
\hline $\mathrm{Q} 30$ & I lie when it's easier than telling the truth & 0.32 & 0.04 & -0.08 & 0.38 \\
\hline Q32 & I lie when telling the truth is too troublesome & 0.32 & $7.61 \mathrm{e}-03$ & -0.15 & 0.38 \\
\hline Q3 & I never tell lies & 0.03 & -0.13 & 0.26 & -0.32 \\
\hline
\end{tabular}

The final version of the LIE questionnaire assesses 4 latent dimensions measured with 4 items each (16 items in total). Based on the most loading items (see Table 2), we labeled these factors Ability (representing one's subjective ability and ease to create and deliver believable lies), Frequency (representing one's subjective and relative assessment of lying frequency), Negativity (the unwillingness to lie related to negative internal factors such as moral values and/or emotional reactions associated with lying) and Contextuality (the flexible and context-driven willingness to lie depending on external factors such as stakes, necessity and alternative options). These factors were significantly correlated together (see Figure 1), with Ability, Frequency and Contextuality showing positive relationships, and Negativity being negatively associated with them. We back-fitted the CFA model on the full dataset in order extract individual factor scores.

In line with recent recommendations, we assessed the multidimensional reliability by means of omega coefficients (Green \& Yang, 2015; Watkins, 2017), suggesting in gen- 


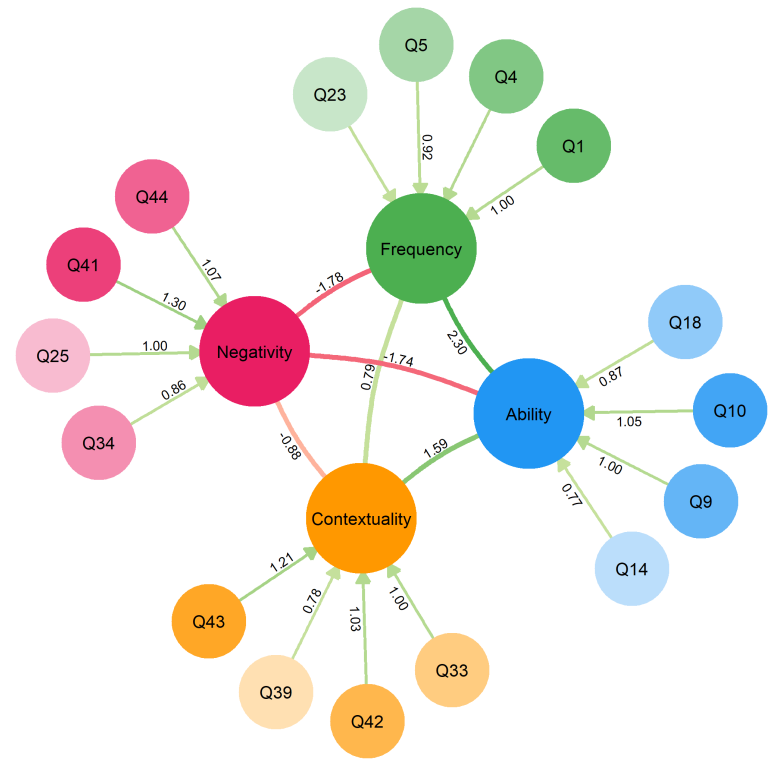

Figure 1. Confirmatory structure of the deception scale items and the correlation between the latent factors. Red links represent negative correlations and green links represent positive correlations. The green arrows represent the loadings of the items onto their respective factors. The numbers correspond to the regression coefficients from the Confirmatory Factor Analysis (CFA).

eral a high reliability of the 16 items $\left(\omega_{\text {total }}=0.83\right)$ as well as for each dimension $\left(\omega_{\text {total }}^{\text {Ability }}=0.87 ; \omega_{\text {total }}^{\text {Frequency }}=0.91\right.$; $\left.\omega_{\text {total }}^{\text {Contextuality }}=0.75 ; \omega_{\text {total }}^{\text {Negativity }}=0.76\right)$. Importantly, the analysis confirmed that the 4 dimensions cannot be considered as only reflecting a unique underlying general factor $\left(\omega_{\text {hierarchical }}=0.36\right)$.

\section{Cluster Structure}

We investigated the presence of higher-density regions in the four-dimensional space of the LIE factor structure. The dataset was deemed suitable for clustering (Hopkins' $\mathrm{H}=$ 0.24 ), and the method agreement procedure (aggregating 28 methods to estimate the optimal number of clusters; see Supplementary Materials 1), supported the existence of 2 $(8 / 28)$ or $3(11 / 28)$ clusters. We then applied k-means clustering, which revealed that grouping the participants in 2 and 3 clusters would account for $44.92 \%$ and $57.58 \%$ of the total variance of the four dimensions of the questionnaire, respectively. Thus, we decided to go ahead with the latter solution and assign each participant to its nearest cluster (see Figure 2), labeling them as Average (41.86\% of the sample; people that report an average lying ability, slightly lower than average frequency, average negativity and contextual- ity), Trickster (35.04\% of the sample; people with high reported lying ability, frequency, low negative experience associated with deception and above-average flexibility in its implementation), and Virtuous (23.10\% of the sample; people with very low reported lying ability and frequency, strong negative emotions and moral attitude associated with lying and high rigidity in their (non-)usage of deception).

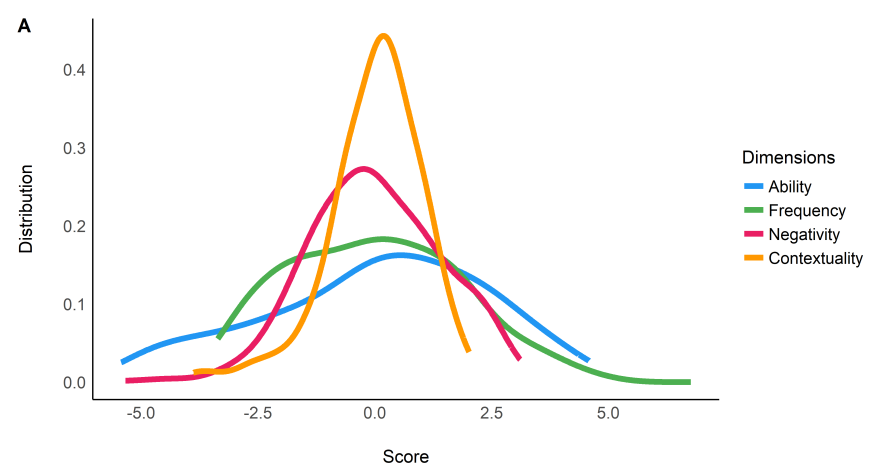

B

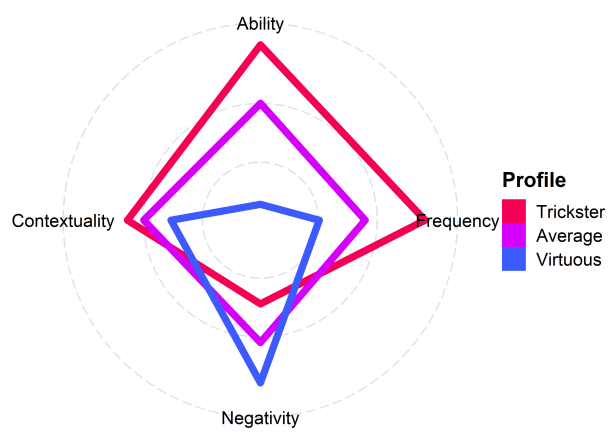

Figure 2. The distribution of the LIE dimensions (A) and the centre values of the 3 clusters of participants.

\section{Convergent Validity}

Bayesian Regressions (from which we will report the $89 \%$ Credible Interval (CI) and the probability of direction $p d$, a Bayesian equivalent of the $p$-value; see Makowski, BenShachar, Chen, et al., 2019), using both the LIE dimensions and profiles were used to assess the role of demographic variables, and Gaussian Graphical Models (GGMs, Epskamp et al., 2018), i.e., networks based on partial correlations, were used to assess the links between the LIE dimensions and other theoretically related constructs. As the details of the analyses are available in Supplementary Materials 1, we will only report in the manuscript the significant links (Bonferroni corrected and $p<.001$ for the GGMs to control for spurious links).

\section{Demographics.}

Sex. 
We fitted two Bayesian logistic regressions to predict Sex with the lying profile (offering a simpler combined and integrated perspective), and the 4 lying dimensions (to assess the underlying driving effects independently of one another). These revealed that men, as opposed to women, were more likely to be Tricksters than Average (coefficient $=0.41,89 \%$ CI [0.13, 0.67], pd $=99.15 \%$ ) as well as more likely to be Average than Virtuous (coefficient $=-0.48,89 \%$ CI $[-0.81$, $-0.19]$, pd $=99.38 \%$ ). This effect was likely to be driven by Ability, the only dimension significantly sensitive to sex (coefficient $=0.14,89 \%$ CI $[0.07,0.21], \mathrm{pd}=99.85 \%)$, reported as higher by men than by women.

Age.

The Bayesian linear mixed models predicting Age were adjusted for Sex (entered as random factor), Income and $E d$ ucation (entered as fixed effects). Age was higher for the Virtuous, relative to the Average profile (coefficient $=2.81$, $89 \%$ CI [1.68, 4.01], pd = 100\%). These differences were likely driven by Ability which was negatively related to Age (coefficient $=-0.36,89 \%$ CI $[-0.60,-0.13], \mathrm{pd}=99.17 \%$ ), suggesting that younger people tend to portray themselves as good liars.

\section{Socio-Economical Status.}

The Bayesian linear mixed models predicting Income were adjusted for Sex (entered as random factor), Education and Age (entered as fixed effects). Although the profiles were not different in terms of Income, Ability was the only dimension significantly and positively related to Income (coefficient $=$ $189.56,89 \%$ CI $[28.94,342.75], \mathrm{pd}=97.12 \%)$.

\section{Education.}

The Bayesian linear mixed models predicting Education were adjusted for Sex (entered as random factor) and Age (entered as fixed effects). Education was higher for the Virtuous $($ coefficient $=0.36,89 \%$ CI $[0.08,0.61], \mathrm{pd}=98.62 \%)$ and lower for Trickster (coefficient $=-0.36,89 \%$ CI $[-0.58$, $-0.12], \mathrm{pd}=99.52 \%$ ), both relative to the Average profile. No dimension was significantly related to Education.

\section{Faith.}

Due to their strong correlation $(\mathrm{r}=0.86, p<.001)$, we collapsed the two religion-related items into one Faith variable. The Bayesian linear mixed models predicting Faith were adjusted for religion type (entered as a random factor). Faith was stronger for the Virtuous, relative to Average profile (coefficient $=0.67,89 \%$ CI $[0.34,1.05], \mathrm{pd}=99.85 \%)$ and weaker for Tricksters relative to the Average (coefficient $=$ $-0.37,89 \%$ CI [-0.68, -0.07], pd $=97.42 \%$ ). This effect was likely driven by the fact that all lying dimensions - except Ability - were associated with Faith. Stronger Faith was related to higher Negativity (coefficient $=0.53,89 \%$ CI $[0.38$, 0.68], pd $=99.98 \%)$, lower Contextuality $($ coefficient $=-0.25$,
$89 \%$ CI [-0.37, -0.12], pd $=99.98 \%)$ and higher Frequency (coefficient $=0.25,89 \%$ CI $[0.14,0.36], \mathrm{pd}=100 \%)$.
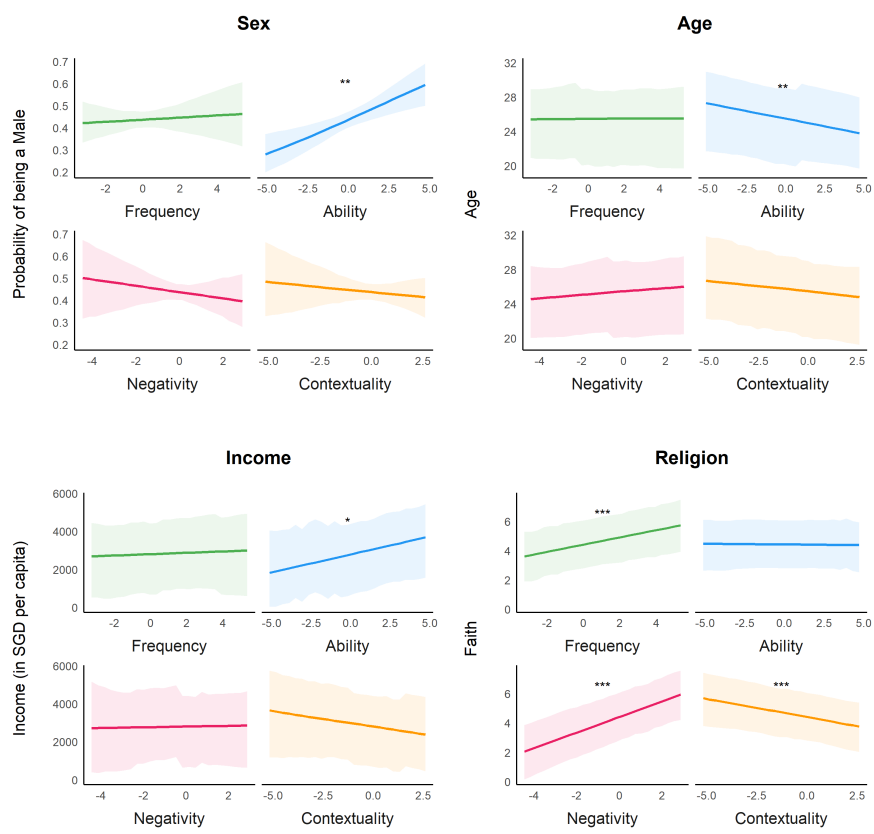

Figure 3. The linear relationship between lying dimensions and demographic variables. Asterisks represent effects with a significant probability of existence $\left({ }^{\circ}>95 \%, *>97 \%, * *>99 \%, * * *>\right.$ $99.9 \%)$.

Absolute Lying Frequency. The Bayesian linear models predicting absolute lying frequency (in lies told per day; adjusted for social desirability) revealed that Tricksters reported a higher (coefficient $=0.51,89 \%$ CI $[0.39,0.64], \mathrm{pd}=$ $100 \%$ ), and the Virtuous a lower (coefficient $=-0.35,89 \% \mathrm{CI}$ $[-0.49,-0.22], \mathrm{pd}=100 \%$ ) absolute lying frequency than the Average, respectively. Specifically, the dimensional model suggested that relative Frequency (coefficient $=0.17,89 \%$ CI $[0.13,0.21]$, pd $=100 \%$ ), as measured by the questionnaire, was significantly predicting the absolute Frequency of lies told per day (see Figure 4).

Social Desirability. The GGM network suggested that Self-Deceptive Enhancement was positively associated with reported lying Ability ( $\mathrm{r}=0.21,95 \% \mathrm{CI}[0.14,0.28]$ ) and negatively with lying Frequency $(\mathrm{r}=-0.20,95 \%$ CI $[-0.27$, -0.13]). On the other hand, active Impression Management was positively associated with Negativity $(\mathrm{r}=0.12,95 \% \mathrm{CI}$ $[0.05,0.19])$ and negatively with Frequency $(\mathrm{r}=-0.13,95 \%$ CI $[-0.20,-0.06])$ and Contextuality $(\mathrm{r}=-0.17,95 \%$ CI [$0.24,-0.10]$ ). The relationship between lying Frequency and Contextuality also changed from positive (in the previous analyses) to negative ( $\mathrm{r}=-0.17,95 \% \mathrm{CI}[-0.23,-0.10])$.

Psychopathy. To avoid contamination of the following models by previously identified mediators, we adjusted LIE 


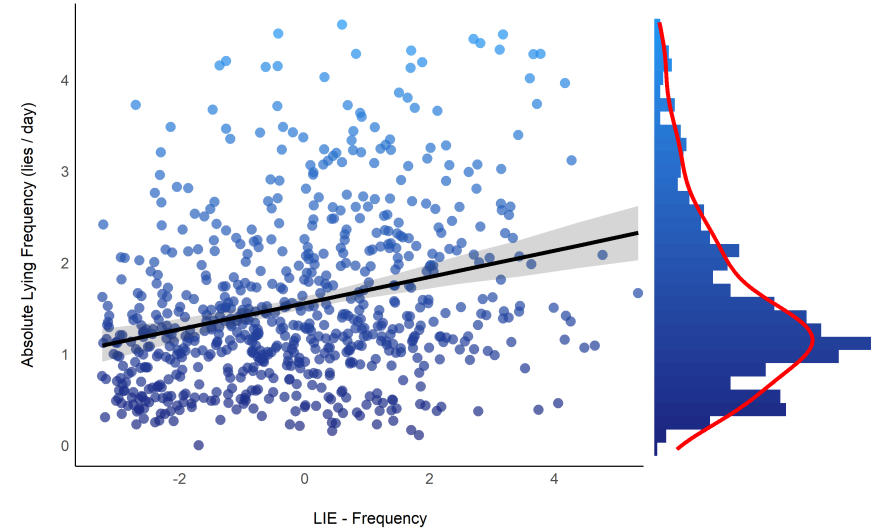

Figure 4. The relationship between the reported absolute frequency (the number of lies told per day, which distribution is showed on the right) and the Frequency dimension of the questionnaire.

scores by regressing out social desirability, age and sex. The GGM network investigating the relationship with psychopathy suggested that lying Ability was positively associated with Boldness ( $\mathrm{r}=0.23,95 \%$ CI [0.16, 0.30]), that Frequency was positively associated with Disinhibition $(r=0.23,95 \%$ CI $[0.17,0.30])$ and that Negativity was negatively associated with Meanness ( $\mathrm{r}=-0.19$, 95\% CI [-0.26, -0.12]) but also positively with Disinhibition $(\mathrm{r}=0.15,95 \%$ CI [0.08, 0.22]).

Narcissism. The GGM network suggested that lying was mainly associated with three core components of narcissism. Lying Ability was positively associated with Manipulativeness $(\mathrm{r}=0.35,95 \%$ CI $[0.29,0.43])$. Negativity was negatively associated with Exploitativeness $(\mathrm{r}=-0.13,95 \% \mathrm{CI}$ $[-0.20,-0.05])$.

Normal Personality. The GGM network suggested that lying was mainly associated with two dimensions of normal personality. Lying Ability was positively associated with Openness ( $\mathrm{r}=0.15,95 \%$ CI [0.08, 0.22]). Lying Negativity was negatively associated with Honesty/Humility ( $\mathrm{r}=-0.15$, 95\% CI [-0.22, -0.08])

Pathological Personality. The GGM network suggested that lying was mainly associated with two dimensions of pathological personality. Lying Ability was positively associated with Antagonism ( $\mathrm{r}=0.19,95 \%$ CI $[0.12,0.26])$ and Frequency was positively associated with Disinhibition $(\mathrm{r}=$ $0.13,95 \%$ CI $[0.05,0.20])$.

Light Triad. The GGM network suggested that lying was independent of the Light Triad facets.

Impulsivity. The GGM network suggested that lying Frequency was positively associated with Positive Urgency $(\mathrm{r}=$ $0.16,95 \%$ CI $[0.09,0.23])$ and that Negativity was negatively associated with the Lack of Premeditation $(\mathrm{r}=-0.15,95 \% \mathrm{CI}$ $[-0.21,-0.08])$.
Emotion Regulation. The GGM network suggested that lying Frequency was positively associated with deficits of Impulse control in emotional contexts $(r=0.13,95 \% \mathrm{CI}$ $[0.05,0.19])$.

Interoception. The GGM network suggested that lying was independent of the sensitivity to bodily signals.

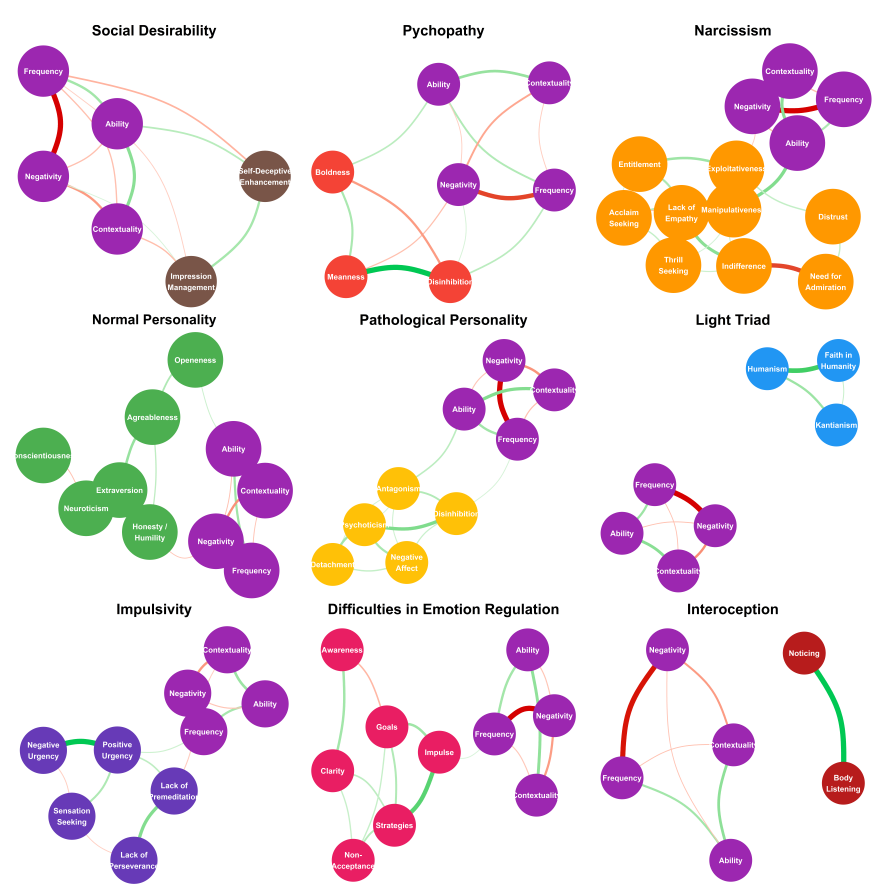

Figure 5. Gaussian Graphical Models (GGMs) for convergent validity with other constructs. The relationships with the lying dimensions were, for all networks except the first, adjusted for social desirability, age and sex. Red and green links represent negative and positive correlations, respectively.

\section{Discussion}

This study aimed at investigating the structure of dispositional deception and its personality correlates by validating a lying questionnaire on a diverse sample. Using a cognitive perspective, we attempted to decompose lying as a highlevel phenomenon, and explore the distinct underlying mechanisms that contribute to it. Our findings suggest that deception, taken as a trait, comprises of four latent dimensions, namely ability (the reported proficiency and ease to create and deliver believable lies), frequency (the reported tendency to lie), negativity (the negative perception of lying related to internal factors, such as emotions or moral values), and contextuality (flexibility of one's willingness to lie related to external factors such as stakes, necessity or alternative options). Although this multi-dimensionality was shown to be robust and reliable, the consistent residual inter-correlation between the four dimensions is nonetheless be compatible 
with the existence of an underlying over-arching general factor. Such general inclination towards lying also manifests in the existence of distinct lying profiles, i.e., clusters of specific patterns of the dimensional structure. This, in turn, underlines the specificity and distinctiveness of lying as a phenomenon, suggesting its conceptualization as a special action and context that requires, triggers and recruits a specific combination of - and interaction between - a specific set of distinct general-purpose mechanisms. In other words, the different facets of lying share a common core component that is specific to its object and purpose. Thus, it appears that deception can hardly be reduced to the sum of its underlying mechanisms, and that a global perspective is required to appropriately capture this phenomenon.

Importantly, we also investigated how these facets of deception are related to other dispositional characteristics. In line with our hypotheses, we found a significant relationship with antisocial traits. Specifically, individuals presenting with antagonistic attributes, such as meanness, boldness and manipulativeness reported high deception abilities, deception frequency and low negativity related to lying. Though the negative relationship between meanness and negativity was unexpected, a likely explanation is that individuals who score high on antisocial traits confer less reliance on moral principles when making the decision to lie or have less negative emotional reactivity towards lying, which translates into greater ease when lying.

We were initially expecting to find the opposite relationship with markers of a benevolent nature. However, under our stringent statistical criteria, we found no significant relationship between deception and pro-social traits. This is in line with recent studies showing a relative independence of benevolence traits in relation to malevolent traits (Kaufman et al., 2019; Tortoriello \& Hart, 2019), or a more complex and subtle pattern of the relationship between the (seemingly) opposite extremes of human nature. For instance, narcissism, traditionally considered as one of the pillars of antisociality, has been found to show an independent, positive correlation to benevolent traits (Kaufman et al., 2019). Aside from further challenging the notion of a clear and relevant dichotomy between so-called "dark" and "light" sides of personality, our findings suggest that a pro-social attitude and nature can co-exist with all types and forms of dispositional deception.

Our study also confirmed links with specific dimensions of normal personality. In particular, lying ability was positively related to one's openness to experiences. This link might be mediated by the increased tendency of exposure to complex, new and uncertain contexts, such as social situations, that would in turn create more opportunities for lying (e.g., for preserving others' impression of themselves), thus nurturing confidence in one's ability to lie. However, we also found honesty-humility to be negatively associated with negative perceptions of lying. Though this link might appear contradictory initially, one possible explanation is that individuals with strong honest-humility traits tend to approach deception with a more neutral stance. They may circumvent any moral judgment that typically comes with it, cooperating genuinely with others even in situations where they have been exploited and/or lied to. Their sincerity and honesty may drive them to perceive deception as an objectively common phenomenon with no intrinsic nor absolute moral value.

One striking finding of the present study is the relationship between specific aspects of dispositional deception and markers of cognitive control (more exactly, proxies of cognitive control deficits). We showed that individuals with difficulties in cognitive control tend to have a higher lying frequency, indicating the involvement of executive functions (e.g., inhibition and flexibility) in the controlled delivery of lies and manipulation of reality. Importantly, this pattern was consistently found across different measures, such as impulsivity, emotion regulation deficits, and disinhibited behavior. Further and more direct investigations of the differential role of executive functions in deception are necessary to cast more direct light on the mechanisms at stake.

Interestingly, a facet of impulsivity, namely the lack of premeditation was inversely related to negative perceptions of lying. These traits implicate one's ability to plan lies systematically and focus their attention on the the task of lying. This result suggests that the ability to consider the emotional and practical implications of one's actions plays a significant role in deception. A greater tendency to weigh the consequences of lying enhances perceptions of negativity towards lying as an act, as lying can be cognitively and emotionally overwhelming. However, while we would have expected such negative perceptions to be more pronounced in individuals with low emotion regulation skills and high interoceptive sensibility, we found no evidence in favor of this hypothesis. Nevertheless, it is important to note that embodied constructs, such as interoceptive and emotion regulation abilities, are only partially measured via self-reported questionnaires (Barrett et al., 2004; Garfinkel et al., 2015). Thus, we suspect that the tools used in the present study were not sufficiently sensitive, or simply not appropriate, which prevents us from drawing any definite conclusions regarding the absence of associations between these dimensions. Future studies should investigate the role of such embodied aspects of cognition through more direct means.

Finally, we emphasize the importance of measuring - and controlling for - social desirability when attempting to measure morally or socially loaded constructs, such as lying, through self-reported questions. Our findings suggest a strong yet subtle relationship, revealing that individuals who perceive themselves more favorably rated themselves as bet- 
ter, yet less frequent, liars. Additionally, people who tend to consciously and actively portray themselves in a socially desirable manner are both more likely to report stronger negativity towards lying and being less influenced by external factors in their decision to lie. In line with this pattern, they also report lying less frequently, which can be observed using different types of measures, such as the absolute frequency of lying (i.e., the number of lies told a day). It is interesting to note that after controlling for social desirability, the relationship between lying frequency and context-driven willingness to lie shifted from positive to negative. Consistent with our other results, we suggest that a common cognitive basis might be driving this relationship. In particular, cognitive control, which allows and supports the control and inhibition over the tendency to lie, would also be necessary for facilitating flexible and context-driven usage. Thus, people who tend to lie a lot in general also use this strategy in a less parsimonious and adaptive manner.

Importantly, we found that lying behavior is also modulated by demographic variables. Consistent with previous findings (Elaad, 2018), individuals who are male and have a higher income reported a higher ability to lie. This concurs with research showing that malevolent traits are negatively associated with being female and income (Kaufman et al., 2019). The age- and sex-related links with honesty, social desirability, and boldness, as well as the relationship between income and self-control (Duckworth, 2011; Moffitt et al., 2011), are likely to mediate such effects.

In addition, we found that people with high religiosity tend to perceive lying as intrinsically negative (possibly related to more absolute or immanent moral values) and are less pressured by the external context necessitating lying (suggesting a stronger role of these moral values). However, our findings also suggest that religiosity is positively correlated with lying frequency. Although surprising, this effect could be mediated by an increased honesty of religious people, as well by the fact that the greater negativity (and therefore saliency) of lies enhances the encoding and ease of retrieval of these events, leading in turn to an overestimation of their frequency.

\section{Limitations and Future Directions}

It is important to note that lies are not all alike and that different types exist, from "harmless" white lies in daily social interactions to more serious and high stakes attempts, for instance to conceal an act of law violation. Moreover, lying is arguably not, in most cases, the end goal but rather the means to attain given goals, which vary extensively across the population. Lying types and motives, although not the focus of the present study, are potentially relevant aspects to gain a comprehensive understanding of this phenomenon. However, one of the main challenges of investigating lying motives is the validation of a robust and usable framework for the classification of lies. For instance, Weber (2017) suggested the existence of 11 types of lies while Zvi and Elaad (2018) emphasized 3 motives (self-gain, altruism, and lying for no reason). Their study further shows that vanity and exhibitionism, two facets of narcissism, were respectively predictive of self-beneficial lies and lying for no reason. Though exploring deception-related motivations is beyond the scope of the present study, these previous findings suggest that the extent of one's motivation to lie (and the type of motive) might be sensitive to personality traits, supporting its relevance within a dispositional approach to deception. This serves as a potential avenue of exploration for studies to further delineate the mechanisms involved in deception.

Another interesting and complementary approach could focus on the reception of lies, rather than how individuals perceive themselves as liars. Prior work suggests that extroverts are more proficient at telling lies as well as detecting lies (Elaad \& Reizer, 2015), and that frequent liars are more likely to perceive themselves as good lie detectors (Zvi \& Elaad, 2018), underlining this issue as a promising topic for investigation.

Though the present study did not aim at study cultural determinants or inter-cultural differences in deception, we also acknowledge the possible role of population-related characteristics in influencing our results. In this context, it is important to highlight that many psychology studies, including task and questionnaire validations, have been criticized for their overrepresentation of a biased subset of participants (referred to as "WEIRD" samples - Western Educated Industrialised Rich and Democratic, Henrich et al., 2010). The demographics of our Singapore sample is not only predominantly nonwestern, but is diverse in terms of age, SES, ethnicity, culture and religion (Singapore being a multicultural and multiethnic society), which constitutes a unique strength of the present study. That said, the possible sample-related specificities warrant extra caution on any claims of generalization, as the conceptualization of lying may vary across cultural norms. In most of deception research using WEIRD samples, where self-interest is at the core of decision-making, lying is perceived as a strategic choice to achieve personal goals (Rodriguez, 1996). On the other hand, members of collectivistic communities are comparatively more motivated by their social obligation to group members (e.g., lying to help other members 'save face'; Gudykunst et al. (1988)) (Rodriguez, 1996). The motivation to lie, and hence the context in which lying occurs, is thus very much dependent on one's cultural identity. As we did not measure specifically culturedependent constructs, we cannot preclude the possibility that a different population may give rise to differences. It is thus critical that future studies investigate intercultural differences and carry out a cross-cultural validation of the structure of 
deception.

Finally, it is crucial to corroborate questionnaire scores with actual behavior (Serota et al., 2010) to support the validity of such self-reported measures. However, behavioral experiments have been lacking in ecological validity due to paradigms that fail to elicit realistic lying from participants in a way that is self-motivated and spontaneous (e.g., participants are sometimes instructed to lie). The use of games with monetary incentives seems to be a promising approach to investigate whether actual lying behaviors are consistent with self-reported measures of lying (Levine et al., 2010).

In conclusion, this study attempted to investigate the traitlike aspect of deception. On a theoretical level, our findings underline lying as a specific yet multi-faceted phenomenon, related to (and modulated by) a variety of inter-individual characteristics. On a practical level, the brief questionnaire validated in this study will allow for including this measure in future experiments on deception to obtain a more complete and accurate picture of its behavioral and neural correlates. Nevertheless, further investigation is warranted to understand how lying behavior varies across different motivating factors, contexts and implications, as well as specific populations defined by factors like culture, pathology, and criminality.

\section{References}

Abe, N. (2009). The neurobiology of deception: Evidence from neuroimaging and loss-offunction studies. Current Opinion in Neurology, 22(6), 594-600. https://doi.org/10.1097/WCO. $0 \mathrm{~b} 013 \mathrm{e} 328332 \mathrm{c} 3 \mathrm{cf}$

Al-Dajani, N., Gralnick, T. M., \& Bagby, R. M. (2016). A psychometric review of the personality inventory for DSM-5 (PID-5): Current status and future directions. Journal of Personality Assessment, 98(1), 62-81.

Aquino, K., \& Becker, T. E. (2005). Lying in negotiations: How individual and situational factors influence the use of neutralization strategies. Journal of Organization Behavior, 21, 661-679.

Arndt, J. E., Hoglund, W. L., \& Fujiwara, E. (2013). Desirable responding mediates the relationship between emotion regulation and anxiety. Personality and Individual Differences, 55(2), 147151.

Ashton, M. C., \& Lee, K. (2009). The HEXACO60: A short measure of the major dimensions of personality. Journal of Personality Assessment, 91(4), 340-345.

Azizli, N., Atkinson, B. E., Baughman, H. M., Chin, K., Vernon, P. A., Harris, E., \& Veselka, L. (2016). Lies and crimes: Dark triad, misconduct, and high-stakes deception. Personality and Individual Differences, 89, 34-39.

Barrett, L. F., Quigley, K. S., Bliss-Moreau, E., \& Aronson, K. R. (2004). Interoceptive sensitivity and self-reports of emotional experience. Journal of Personality and Social Psychology, 87(5), 684.

Bechara, A., \& Damasio, A. R. (2005). The somatic marker hypothesis: A neural theory of economic decision. Games and Economic Behavior, 52(2), 336-372.

Bond, C. F., \& Robinson, M. (1988). The evolution of deception. Journal of Nonverbal Behavior, 12(4), 295-307.

Carlson, S. M., \& Wang, T. S. (2007). Inhibitory control and emotion regulation in preschool children. Cognitive Development, 22(4), 489510 .

Christ, S. E., Van Essen, D. C., Watson, J. M., Brubaker, L. E., \& McDermott, K. B. (Oxford 
University Press, UK). The contributions of prefrontal cortex and executive control to deceptoin: Evidence from activatoin likelihood estimate meta-analyses. Cerebral Cortex, 19(7), 1557-1566.

Cyders, M. A., Littlefield, A. K., Coffey, S., \& Karyadi, K. A. (2014). Examination of a short english version of the UPPS-p impulsive behaviour scale. Addictive Behaviours, 70, 13721376.

Debey, E., Verschuere, B., \& Crombez, G. (2012). Lying and executive control: An experimental investigation using ego depletion and goal neglect. Acta Psychologica, 140(2), 133-141.

DePaulo, B. M., Kashy, D. A., Kirkendol, S. E., Wyer, M. M., \& Epstein, J. A. (1996). Lying in everyday life. Journal of Personality and Social Psychology, 70(5), 979.

Dike, C. C., Baranoski, M., \& Griffith, E. E. H. (2005). Pathological lying revisited. The Journal of the American Academy of Psychiatry and the Law Online, 33(3), 342-349.

Duckworth, A. L. (2011). The significance of selfcontrol. Proceedings of the National Academy of Sciences, 108(7), 2639-2640.

Dunn, B. D., Galton, H. C., Morgan, R., Evans, D., Oliver, C., Meyer, M., Cusack, R., Lawrence, A. D., \& Dalgleish, T. (2010). Listening to your heart: How interoception shapes emotion experience and intuitive decision making. Psychological Science, 21(12), 1835-1844.

El Haj, M., Saloppé, X., \& Nandrino, J. L. (2018). Destination memory and deception: When i lie to barack obama about the moon. Psychological Research, 82(3), 600-606.

Elaad, E. (2018). Personality, demographic, and psychophysiological correlates of people's selfassessed lying abilities. In Detecting Concealed Information and Deception: Recent Developments (pp. 353-376). https://doi.org/10.1016/ B978-0-12-812729-2.00015-X

Elaad, E., \& Reizer, A. (2015). Personality correlates of the self-assessed abilities to tell and detect lies, tell truths, and believe others. Journal of Individual Differences.

Enticott, P. G., Ogloff, J. R. P., \& Bradshaw, J. L. (2006). Associations between laboratory measures of executive inhibitory control and selfreported impulsivity. Personality and Individual
Differences, 41(2), 285-294. https://doi.org/10. 1016/j.paid.2006.01.011

Epskamp, S., Borsboom, D., \& Fried, E. I. (2018). Estimating psychological networks and their accuracy: A tutorial paper. Behavior Research Methods, 50(1), 195-212.

Eysenck, H. J., \& Eysenck, S. B. G. (1975). Manual of the eysenck personality questionnaire (junior and adult). Hodder; Stoughton.

Fino, E., Melogno, S., Iliceto, P., D’Aliesio, S., Pinto, M. A., Candilera, G., \& Sabatello, U. (2014). Executive functions, impulsivity, and inhibitory control in adolescents: A structural equation model. Advances in Cognitive Psychology, 10(2), 32.

Fisher, R. J., \& Katz, J. E. (2000). Socialdesirability bias and the validity of self-reported values. Psychology \& Marketing, 17(2), 105120.

Fullam, R. S., McKie, S., \& Dolan, M. C. (2009). Psychopathic traits and deception: Functional magnetic resonance imaging study. The British Journal of Psychiatry, 194(3), 229-235.

Furman, D. J., Waugh, C. E., Bhattacharjee, K., Thompson, R. J., \& Gotlib, I. H. (2013). Interoceptive awareness, positive affect, and decision making in major depressive disorder. Journal of Affective Disorders, 151(2), 780-785.

Füstös, J., Gramann, K., Herbert, B. M., \& Pollatos, O. (2012). On the embodiment of emotion regulation: Interoceptive awareness facilitates reappraisal. Social Cognitive and Affective Neuroscience, 8(8), 911-917. https://doi.org/10.1093/ scan/nss089

Garfinkel, S. N., Seth, A. K., Barrett, A. B., Suzuki, K., \& Critchley, H. D. (2015). Knowing your own heart: Distinguishing interoceptive accuracy from interoceptive awareness. Biological Psychology, 104, 65-74.

Gillath, O., Sesko, A. K., Shaver, P. R., \& Chun, D. S. (2010). Attachment, authenticity, and honesty: Dispositional and experimentally induced security can reduce self-and other-deception. Journal of Personality and Social Psychology, 98(5), 841.

Gozna, L. F., Vrij, A., \& Bull, R. (2001). The impact of individual differences on perceptions of lying in everyday life and in a high stake situ- 
ation. Personality and Individual Differences, 31(7), 1203-1216.

Green, S. B., \& Yang, Y. (2015). Evaluation of dimensionality in the assessment of internal consistency reliability: Coefficient alpha and omega coefficients. Educational Measurement: Issues and Practice, 34(4), 14-20.

Gudykunst, W. B., Ting-Toomey, S., \& Chua, E. (1988). Culture and interpersonal communication. Sage Publications, Inc.

Halevy, R., Shalvi, S., \& Verschuere, B. (2014b). Being honest about dishonesty: Correlating self-reports and actual lying. Human Соттиnication Research, 40(1), 54-72.

Halevy, R., Shalvi, S., \& Verschuere, B. (2014a). Being honest about dishonesty: Correlating self-reports and actual lying. Human Соттиnication Research, 40(1), 54-72.

Hare, R. D., \& Forth, A. E. (1985). Psychopathy and lateral preference. Journal of Abnormal Psychology, 94(4), 541-546.

Hart, C. M., Ritchie, T. D., Hepper, E. G., \& Gebauer, J. E. (2015). The balanced inventory of desirable responding short form (BIDR-16). SAGE Open, 5(4), 1-9.

Henrich, J., Heine, S. J., \& Norenzayan, A. (2010). Beyond WEIRD: Towards a broad-based behavioral science. Behavioral and Brain Sciences, 33(2-3), 111-135. https://doi.org/10. $1017 / \mathrm{s} 0140525 \times 10000725$

Hirata, S. (1986). Tactical deception and understanding of others in chimpanzees. In $\mathrm{Cog}_{-}$ nitive development in chimpanzees (pp. 265276). Springer-Verlag. https://doi.org/10.1007/ 4-431-30248-4_17

Hopwood, C. J., Thomas, K. M., Markon, K. E., Wright, A. G., \& Krueger, R. F. (2012). DSM5 personality traits and DSM-IV personality disorders. Journal of Abnormal Psychology, 121(2), 424.

Jensen, L. A., Arnett, J. J., Feldman, S. S., \& Cauffman, E. (2004). The right to do wrong: Lying to parents among adolescents and emerging adults. Journal of Youth and Adolescence, 33(2), 101112.

Jonason, P. K., Lyons, M., Baughman, H. M., \& Vernon, P. A. (2014). What a tangled web we weave: The dark triad traits and deception. Personality and Individual Differences, 70(6), 117119.

Jonason, P. K., \& Tost, J. (2010). I just cannot control myself: The dark triad and self-control. Personality and Individual Differences, 49(6), 611615.

Karim, A. A., Schneider, M., Lotze, M., Veit, R., Sauseng, P., Braun, C., \& Birbaumer, N. (2010). The truth about lying: Inhibition of the anterior prefrontal cortex improves deceptive behaviour. Cerebral Cortex, 20(1), 205-213.

Kashy, D. A., \& DePaulo, B. M. (1996). Who lies? Journal of Personality and Social Psychology, 70(5), 1037.

Kaufman, S. B., Yaden, D. B., Hyde, E., \& E, T. (2019). The light vs. Dark triad of personality: Contrasting two very different profiles of human nature. Frontiers in Psychology, 10(467).

Kever, A., Pollatos, O., Vermeulen, N., \& Grynberg, D. (2015). Interoceptive sensitivity facilitates both antecedent- and response-focused emotion regulation strategies. Personality and Individual Differences, 87, 20-23. https://doi.org/10.1016/ j.paid.2015.07.014

Kohn, N., Eickhoff, S. B., Scheller, M., Laird, A. R., Fox, P. T., \& Habel, U. (2014). Neural network of cognitive emotion regulation an ALE meta-analysis and MACM analysis. NeuroImage, 87, 345-355. https://doi.org/10.1016/ j.neuroimage.2013.11.001

Lee, T. M., Au, R. K., Liu, H.-L., Ting, K., Huang, C.-M., \& Chan, C. C. (2009). Are errors differentiable from deceptive responses when feigning memory impairment? An fMRI study. Brain and Cognition, 69(2), 406-412.

Leiner, D. J. (2013). Too fast, too straight, too weird: Post hoc identification of meaningless data in internet surveys. SSRN Electronic Journal. https://doi.org/10.2139/ssrn.2361661

Levine, T. R., Kim, R. K., \& Hamel, L. M. (2010). People lie for a reason: Three experiments documenting the principle of veracity. Communication Research Reports, 27(4), 271-285.

Lüdecke, D., Makowski, D., \& Waggoner, P. (2019). Performance: Assessment of regression models performance. $R$ Package Version 0.4, 2.

Lüdecke, Daniel, Waggoner, P., \& Makowski, D. (2019). Insight: A unified interface to access 
information from model objects in r. Journal of Open Source Software, 4(38), 1412. https: //doi.org/10.21105/joss.01412

Makowski, D. (2018). Cognitive neuropsychology of implicit emotion regulation through fictional reappraisal $[\mathrm{PhD}$ thesis, Universite Sorbonne Paris Cité]. http://www.theses.fr/s176981

Makowski, D., Ben-Shachar, M., \& Lüdecke, D. (2019a). bayestestR: Describing Effects and their Uncertainty, Existence and Significance within the Bayesian Framework. Journal of Open Source Software, 4(40), 1541. https://doi. org/10.21105/joss.01541

Makowski, D., Ben-Shachar, M. S., Chen, S. A., \& Lüdecke, D. (2019). Indices of effect existence and significance in the bayesian framework. https://doi.org/10.31234/osf.io/2zexr

Makowski, D., Ben-Shachar, M. S., \& Lüdecke, D. (2019b). Describe and understand your model's parameters. CRAN. https://github.com/ easystats/parameters

Makowski, D., Sperduti, M., Lavallée, S., Nicolas, S., \& Piolino, P. (2019). Adaptation and validation of a short french version of the affective style questionnaire. International Journal of Cognitive Therapy.

Makowski, D., Sperduti, M., Nicolas, S., \& Piolino, P. (2017). "Being there" and remembering it: Presence improves memory encoding. Consciousness and Cognition, 53, 194-202. https: //doi.org/10.1016/j.concog.2017.06.015

Mehling, W. E., Acree, M., Stewart, A., Silas, J., \& Jones, A. (2018). The multidimensional assessment of interoceptive awareness, version 2 (MAIA-2). PloS One, 13(12), e0208034.

Miller, J. D., Few, L. R., Wilson, L., Gentile, B., Widiger, T. A., MacKillop, J., \& Keith Campbell, W. (2013). The five-factor narcissism inventory (FFNI): A test of the convergent, discriminant, and incremental validity of FFNI scores in clinical and community samples. Psychological Assessment, 25(3), 748-758.

Moffitt, T. E., Arseneault, L., Belsky, D., Dickson, N., Hancox, R. J., Harrington, H., Houts, R., Poulton, R., Roberts, B. W., Ross, S., \& others. (2011). A gradient of childhood self-control predicts health, wealth, and public safety. Proceedings of the National Academy of Sciences, 108(7), 2693-2698.
Ochsner, K. N., \& Gross, J. J. (2005). The cognitive control of emotion. Trends in Cognitive Sciences, 9(5), 242-249.

Panasiti, M. S., Pavone, E. F., Merla, A., \& Aglioti, S. M. (2011). Situational and dispositional determinants of intentional deceiving. PloS One, 6(4), e19465.

Patrick, Christopher J. (2010). Triarchic psychopathy measure. American Psychological Association (APA). https://doi.org/10.1037/t42471-000

Patrick, Christopher J., Fowles, D. C., \& Krueger, R. F. (2009). Triarchic conceptualization of psychopathy: Developmental origins of disinhibition, boldness, and meanness. Development and Psychopathology, 21(3), 913-938.

Paulhus, Delroy L. (1991). Measurement and control of response bias. In Measures of personality and social psychological attitudes (pp. 1759). Elsevier. https://doi.org/10.1016/b978-012-590241-0.50006- $\mathrm{x}$

Paulhus, Delroy L., \& Williams, K. M. (2002). The dark triad of personality: Narcissism, machiavellianism and psychopathy. Journal of Research in Personality, 36(6), 556-563.

Poletti, M., Borelli, P., \& Bonuccelli, U. (2011). The neuropsychological correlates of pathological lying: Evidence from behavioral variant frontotemporal dementia. Journal of Neurology, 258(11), 2009-2013. https://doi.org/10.1007/ s00415-011-6058-1

R Core Team. (2019). R: A language and environment for statistical computing. R Foundation for Statistical Computing. https://www.Rproject.org/

Revelle, W. (2018). Psych: Procedures for psychological, psychometric, and personality research. Northwestern University. https:// CRAN.R-project.org/package=psych

Riva, G., Mantovani, F., Capideville, C. S., Preziosa, A., Morganti, F., Villani, D., Gaggioli, A., Botella, C., \& Alcañiz, M. (2007). Affective interactions using virtual reality: The link between presence and emotions. CyberPsychology \& Behavior, 10(1), 45-56. https://doi.org/ 10.1089/cpb.2006.9993

Rodriguez, J. (1996). Deceptive communication from collectivistic and individualistic perspectives. Intercultural Communication Studies, 6(2). 
Rosseel, Y. (2012). Lavaan: AnRPackage for structural equation modeling. Journal of Statistical Software, 48(2). https://doi.org/10.18637/ jss.v048.i02

Serota, K. B., \& Levine, T. R. (2015). A few prolific liars: Variation in the prevalence of lying. Journal of Language and Social Psychology, 34(2), $138-157$.

Serota, K. B., Levine, T. R., \& Boster, F. J. (2010). The prevalence of lying in america: Three studies of self-reported lies. Human Communication Research, 36(1), 2-25.

Seth, A. K., Suzuki, K., \& Critchley, H. D. (2012). An interoceptive predictive coding model of conscious presence. Frontiers in Psychology, 2, 395.

Sherman, E. D., Miller, J. D., Few, L. R., Keith Campbell, W., Widiger, T. A., Crego, C., \& Lynam, D. R. (2015). Development of a short form of the five-factor narcissism inventory: The FFNI-SF. Psychological Assessment, 27(3), 1110-1116.

Sibley, C. G., Luyten, N., Purnomo, M., Mobberley, A., Wootton, L. W., Hammond, M. D., Sengupta, N., Perry, R., \& West-Newman, T. (2011). The mini-IPIP6: Validation and extension of a short measure of the big-six factors of personality in new zealand. New Zealand Journal of Psychology, 40(3), 142-159.

Sperduti, M., Makowski, D., Arcangeli, M., Wantzen, P., Zalla, T., Lemaire, S., Dokic, J., Pelletier, J., \& Piolino, P. (2017). The distinctive role of executive functions in implicit emotion regulation. Acta Psychologica, 173, 13-20. https://doi.org/10.1016/j.actpsy.2016.12.001

Tortoriello, G. K., \& Hart, W. (2019). Blurring the dichotomy of good and evil: The idiosyncratic helping strategies associated with unmitigatedagentic and unmitigated-communal personalities. European Journal of Personality.

Turck, M. A. de, \& Miller, G. R. (1985). Deception and arousal: Isolating the behavioral correlates of deception. Human Communication Research, 12(2), 181-201.

Uziel, L. (2014). Impression management ("lie") scales are associated with interpersonally oriented self-control, not other-deception. Journal of Personality, 82(3), 200-212.
Victor, S. E., \& Klonsky, E. D. (2016). Validation of a brief version of the difficulties in emotion regulation scale (DERS-18) in five samples. Journal of Psychopathology and Behavioural Assessment, 38(4), 582-589.

Vrij, A., \& Semin, G. R. (1996). Lie experts' beliefs about non-verbal indicators of deception. Journal of Nonverbal Behavior, 20(1).

Waal, F. B. M. de. (2005). Intentional deception in primates. Evolutionary Anthropology: Issues, News, and Reviews, 1(3), 86-92. https: //doi.org/10.1002/evan.1360010306

Watkins, M. W. (2017). The reliability of multidimensional neuropsychological measures: From alpha to omega. The Clinical Neuropsychologist, 31(6-7), 1113-1126.

Weber, J. T. (2017). When deception gets personal: An exploration into personality's link to deception.

Weiss, B., \& Feldman, R. S. (2006). Looking good and lying to do it: Deception as an impression management strategy in job interviews. Journal of Applied Social Psychology, 36(4), 295-317.

Whiteside, S. P. H., \& Lynam, D. R. (2001). The five factor model and impulsivity: Using a structural model of personality to understand impulsivity. Personality and Individual Differences, 30(4), 669-689.

Wright, G. R. T., Berry, C. J., Catmur, C., \& Bird, G. (2015). Good liars are neither 'dark' nor selfdeceptive. PLOS ONE, 10(6), e0127315.

Zvi, L., \& Elaad, E. (2018). Correlates of narcissism, self-reported lies, and self-assessed abilities to tell and detect lies, tell truths, and believe others. Journal of Investigative Psychology and Offender Profiling, 15(3), 271-286. 